\title{
BioMuse to Bondage: Corporeal Interaction in Performance and Exhibition
}

\author{
Atau Tanaka
}

\section{Introduction}

This chapter looks at technologies of biosensing and techniques of sonification that invoke physicalization of sound to create a sense of intimacy in musical performance and gallery exhibition. By comparing the dynamic across stage and gallery settings, we will see how the corporeal activation of sound can become a key not just to decode experimental performance, but as an entry point to possible intimate spaces created by digital interaction. We will draw upon notions of physicalization, enaction, and embodiment to describe how a visceral, nearly carnal situation can be created in technology mediated work.

Physicalization of events is the translation and representation of phenomena that may be intangible in material, haptic terms. This can take place through natural processes of media transduction to artificial methods of data transcoding. Data visualization is a form of physicalization in which abstract information is rendered physical through graphical representation and visual interpretation. Sonification does the same with acoustical events as the output medium. The inverse is also possible, where sound is visualized or rendered tangible.

Enactive interaction is a research area of human-machine interaction where understanding of a technology system is based on multisensory input and motor responses resulting from active forms of engagement [Enactive]. This approach is built on psychologist Jerome Bruner's notion of enactive knowledge, a form of knowledge organization based on lived experience as distinct from cultural and representational forms of iconic and symbolic knowledge [Bruner 1966]. From this, cognitive scientist Francisco Varela conceived enactive forms of cognition that are rooted in biological systems and their relationship to the environment [Varela 1999]. This results in a embodied view of consciousness and lived experience.

In the area of human-computer interaction research (HCI), Dourish draws upon the philosophy of phenomenology to establish a definition of embodiment for interaction design [Dourish 2004]. He extends the concept of embodiment from an intuitive notion of the physical manifestation to explore its deeper implications in tangible and social interaction. Embodiment denotes not only the setting in which things occur, but expresses activity in concrete, and not purely abstract terms. Entities interact in forms where they can play a range of roles in interaction. He draws upon the history and evolution of phenomenological thought from Brentano, Husserl, and Schutz to Heidegger and Merleau-Ponty, to unwrap concepts of intentionality, objects of consciousness and mental experience (noema and noesis), intersubjectivity, and situated perception. He cites Heidegger's notions of ready-to-hand, that of acting through a transparent entity, and present-at-hand where an entity becomes a formidable focus of conscious attention, and applies them to establishing models for embodied technology interaction. 
We apply the notion of embodied interaction to look at two works, one performance, one exhibition, and use the performative nature of the first to understand the physical sense of the second. Through this reading of the work we will see that both share the dichotomy of being highly visceral yet hands-off and free of direct contact. That this conception can apply across presentation formats of stage and art gallery point to the possible generalization of this approach to expose the intimate potential of digital media.

\section{BioMuse}

The BioMuse is a musical instrument based on physiological biosensing technology. A series of gold plated dry electrodes make electrical contact with the skin, and detect electrical activity from neuron impulses of the body. In this way, cerebral activity (electroencephalogram, EEG), cardioid activity (electrocardiogram, ECG), and muscular activity (electromyogram, EMG) are sensed and digitized, and become human interface data for the articulation of computer processes and media such as digital audio, video, and computer graphics.

My use of the BioMuse has focused on voluntary contraction of forearm muscles to articulate musical gesture [Lusted and Knapp 1996]. This use of the BioMuse in effect turns the performer's body into musical instrument. Through this I have established a performance practice across a range of musical contexts, from solo to ensemble groupings [Bongers 1998] [Tanaka 1993] [Tanaka 2008].

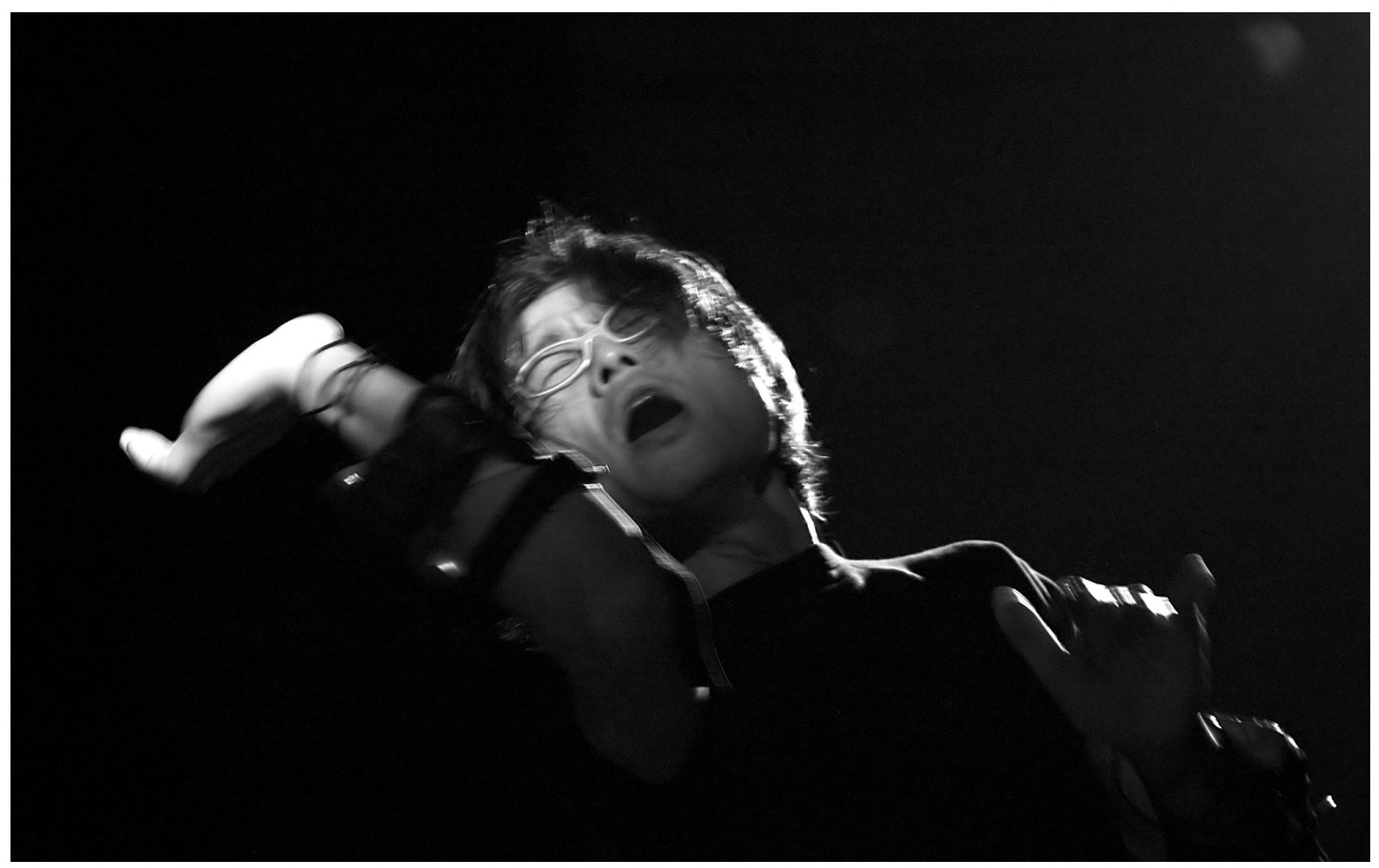

Fig. 1. The BioMuse in concert at STEIM, Amsterdam, 2007. Photo credit: Frank Baldé

The proximity of sensing to the performer's body point to the visceral nature and intimate potential of the BioMuse. This differentiates the BioMuse from other interactive systems where sensors are external to the subject. External sensors such as accelerometers are effective in detecting gestural output that is the result of an action. Through this, rich forms of enactive interaction have been conceived [Di Paolo 2010]. Biosensing, on the other hand, by detecting the physiological signal at 
the origin of an action, captures gestural input, or intention. This intention may or may not manifest itself in legible media output, creating space for ambiguity and interpretation, and within this, the fragile fleeting hints of intimacy.

The space in between performer intention and spectator legibility put in place a space of mutual engagement beyond the typical user oriented focus of enactive interaction. In this regard, we return to the root of enaction as an embodied way to establish a relationship between a subject and the world within which it exists [Varela 1999]. In this case a dynamic is established between the performer and her immediate environment, an audience group, through which subject-centric sense of self, of proprioception, may or may not be communicated to the broader group. The subject/ world relationship in this case is not an externally focused form of enactive knowledge, that of learning about ones world through actions, but rather an internally focused form of intimate knowledge of a subject's state, sensation, and intention, by a public community gained through an empathic reading of corporeal action.

How is this intimate sense of performer state transmitted through sensor interfaces and digital sound? I argue that successful and artful transmission of intimacy in a performance situation requires two elements, first a close proprioceptive loop within the performer himself, and second, a medium for transmitting to the viewer, and immersing him in, an abstraction of the original sensation. To realize this with dematerialized digital media is a challenge that is the focus of the work presented here.

While the BioMuse is highly sensitive in detecting corporeal intention, it is ultimately just a sensing device - that is to say it provides input modality without providing an output mode. While it can detect the performer's actions, it gives nothing back to the performer in terms of resistance, feedback or display. Seen in this light, it is fundamentally different from a physical object that is manipulable. As it is the performer's own body that supposedly becomes musical instrument, there is not any external object, no separate instrument that resonates or offers resistance back to the instrumentalist. In this sense, the system exhibits no haptic feedback.

Without tangible interaction, the BioMuse is not able to provide a traditional channel of proprioception. Without external referents, the performer does not have habitual cues to situate her own body with respect to the world around her or to receive information about the effort she is exerting. At the same time, knowing that the system tracks muscle tension causes the performer to focus on concentrated gesture. Refinement of gesture typically comes gradually as the performer begins to understand what part of the sound they are affecting - when they understand the parameter mapping to sound synthesis. Until this point, a performer getting accustomed to a new interaction tends to exaggerate gesture in search of the effect of their movement on the sound output.

Once an understanding of the mapping of gesture to sound is established, performer movements tend to become more focused and fluid. However it remains at a cerebral, distant level of action and response. This could be considered an example of Bruner's symbolic knowledge [Bruner 1966] where the performer prematurely searches for representational translations of movement to resulting sound. If the gesture/sound relationship evokes a culturally familiar sign, it may fall in the domain of iconic knowledge where mental images shared across people facilitate communication . In Kendon's study of utterance and gesture in human communication, these forms of articulation remain semantic [Kendon 2004]. These are higher level abstract forms of mental modeling that, in 
our case of sonic exploration and performance, cannot be broached until a lower level enactive knowledge is established connecting corporeal action to resulting sound. The visceral, intimate quality are not automatic in a system of this sort, and are necessary before gestural input and sound output can be meaningfully coupled to create music.

\section{Physicalization and psychoacoustics}

It is with the physicalization of sound that we are able to close the haptic loop for the performer and create an empathic, intimate dynamic for the spectator. Physicalizing is used in fields ranging from theatre [Spolin 1999] to product design [Bishop 2009] as a means of representation of intangible entities. Sound can be materialized through automatic processes of scientific visualization [Misra 2005] or of creative transduction of sound into physical phenomena [De Broek and De Mey 2011]. Here the objective is less about translation or representation, but the recognition of sound, however immaterial, as physical phenomena, and approaches to exposing the physical nature of acoustical properties to render musical interaction tangible. Carter introduces the notion of material thinking as forms of discourse and mechanisms of collaboration [Carter 2004]. Here the physicality of material is used not as a collaborative channel between creative entities, but an empathic channel of communicating intimate experience.

In order to most effectively exploit the perceptual potential of physicalized sound, we turn to our knowledge of auditory perception mechanisms of human hearing [Helmholtz 1954/1877] and compose directly as a function of these psychophysical phenomena.

Auditory perception operates at physical, physiological, and psychoacoustic levels. At the most basic, physical level, audio amplitude and selective frequency usage can be used to render sound materialistic. This includes use of amplitude that is sufficient enough, and at frequencies at multiples of low frequency standing wave resonances of physical space, to create a palpable sensation from acoustical pressure waves. Volumetric resonance of physical space can enter an interaction with resonant properties of the human body through bone conduction. The physical distance between the left and right ear establish frequencies where detuning can create a sense of disorientation.

Human hearing is highly sensitive to acoustical phenomena as a way of gauging motion and space [Pierce 1992]. The distance of a sound source is detected in the simple balance between direct sound and reverberated sound. The lateral movement and speed of a moving sound source creates a shifting frequency of perceived sound based on the speed of sound through space to create the Doppler Effect, most commonly heard in everyday life with passing vehicles and fast moving sirens.

The physics of the inner ear are the physiological bases of auditory perception [Levitin 2008], where a series of cues of sound properties are established, and can be manipulated to create auditory illusions. These include the Fletcher Munson curve of amplitude dependent frequency response of human hearing [Fletcher and Munson 1933] in perceptions of loudness. The just-noticeabledifference (JND) in time and in frequency aid in distinguishing multiple sound sources from single sounds. The transition zones in each can be exploited to create ambiguity - the roughness in detuned sound in between out-of-tune beating and clearly distinct notes, and the buzziness of flammed sound in between a smeared single event and two temporally distinct impulses. 
By creating mappings of performance gesture to aspects of sound synthesis that play directly on these perceptual mechanisms, we are able to create physical interaction with an otherwise intangible medium. The feedback loop is complete, as the body on which these mechanisms act are the same body that produce the sound in question. In the simplest case, if muscle tension in the forearm can modulate the frequency and intensity of sound that is reproduced at sufficient amplitude to cause resonant vibration in the bone under that muscle, we have created a form of haptic feedback in the absence of traditional physical objects. In a more complex scenario, as muscle tension varies the frequency distribution of sound in relation to the Fletcher Munson curse, physical effort can be linked to perceived sound power. Similarly with distance cues, performer exertion can literally (or figuratively) push and pull virtual sonic objects in space.

The perceived material relationship to sound can create musical interaction to replace the traditional sensation gained from the manipulation of real physical objects such as acoustic musical instruments. This brings a physicality to sonic interaction that gives a psychomotor satisfaction superior to that achieved or missed, grasping in thin air. This renders tangible the relationship between two otherwise immaterial entities: free space gesture and digital audio. It creates haptic feedback reminiscent of physical objects and brings a visceral dimension of effort and exertion into the articulation of computer generated sound.

This form of haptic feedback is unique in that is operates not through direct object object contact, but across physical, acoustical space. In a concert performance situation, this feedback channel traverses the public space which the performer shares with the audience. The highly personal, and intimate, corporeal sensation experienced by the performer connecting gesture to physicalized sound are thus opened up for outsiders, the listeners, to perceive. With successful sound projection and auditory display, the audience will feel the same sensations in the sound as the performer - the physical vibrations of bones and body, and the psychoacoustic sensations of sound intensity, location, movement, and distance. But instead of being the source of the gesture that produces these material sonic results, the audience sees the performer's actions and effort in direct correlation with their own perceived sensation. If successfully articulated, this creates forms of empathic resonance on the part of the spectator, to sympathize with the performer's effort and to feel themselves what it would be like to produce physical sound in this way. This is a direct, physical understanding that operates at a carnally more basic level than symbolic and iconic knowledge, and accesses Bruner's enactive knowledge.

\section{Bondage}

With Bondage, I sought to transfer the experience of visceral intimacy from a musical performance setting to a gallery exhibition setting. Instead of the audience having the opportunity to get a glimpse into the intimate corporeal experience of a performer through the relationship of gesture and physicalized sound, I used the same devices of tangible sonic perception to heighten a visual art experience, bringing the gallery viewer into intimate contact with the subject of a provocative photograph.

Bondage is an interactive installation work that is based on a Polaroid by the Japanese photographer Nobuyoshi Araki, of a subject in ritualized bondage. The photograph is used as source material and input for a process of sonification, where luminosity in the image is read as sonograph by a computer process that resynthesizes a sound frequency spectrum from the image [Tanaka 2011]. The process is dynamic, as an infrared camera detects presence of viewers in front of the work, and applies a luminance key on the image, exposing the positive of what was otherwise the negative 
projection of the original photograph. This interplay puts the body of the viewer in an abstract, visual relationship with the subject of the Polaroid. The interaction results in a shifting frequency spectrum, heard as continuously evolving soundscape in the exhibition space.

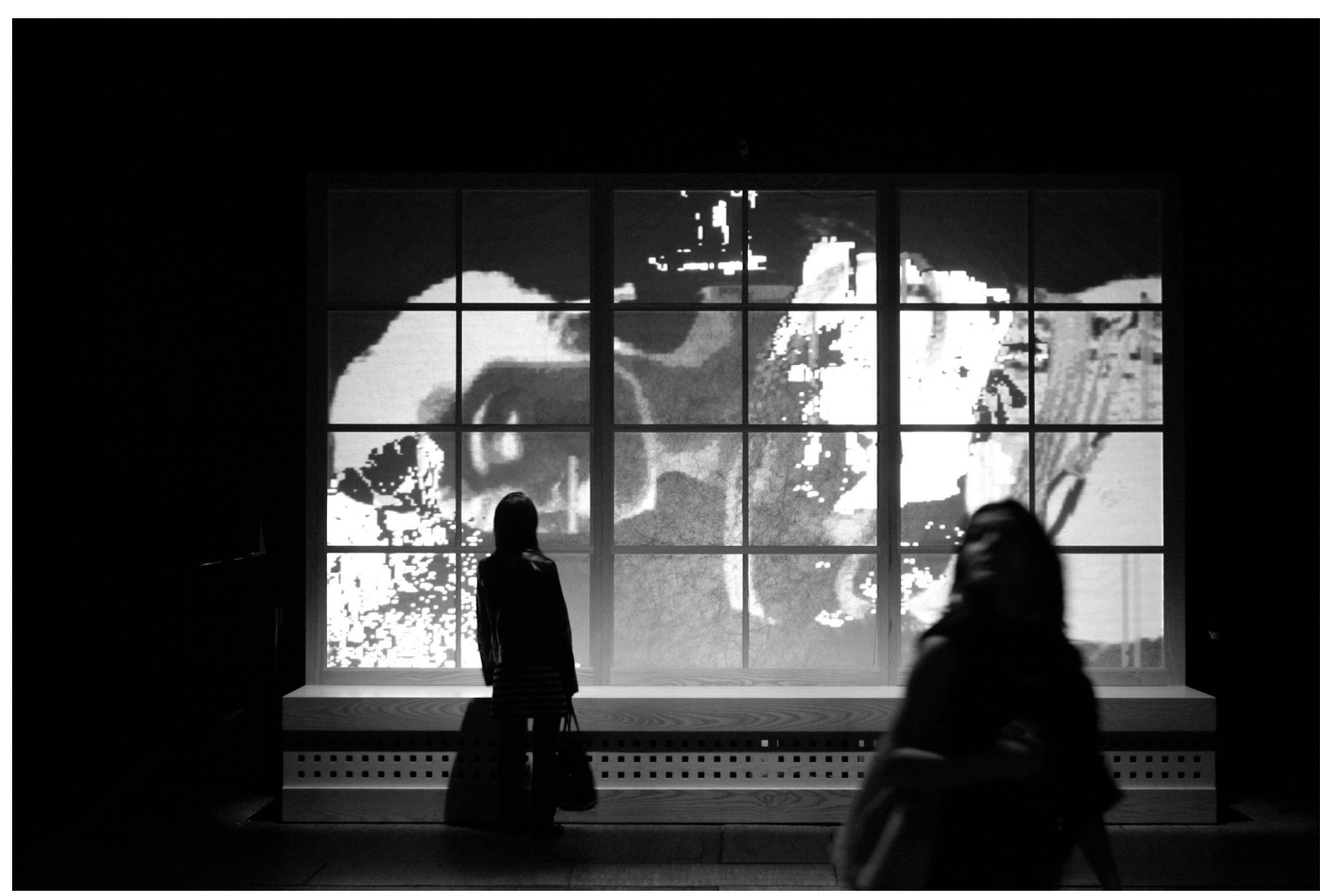

Fig 2. Bondage, exhibited at Villette Numérique, Paris, 2004. Photo credit: Pierre-Emmanuel Rastoin

The original photograph, and its use in exhibition is richly imbued in Japanese cultural iconography. Araki is a master exploiting Japanese imagery, with his subjects dressed in kimonos and sadomasochistic act and action staged in traditional tatami rooms. I sought to extend this already potent imagery by constructing a projection surface that resembled the Japanese shoji panel of paper and wood. The interaction of gallery viewer and photograph, the spontaneous dance of the spectator's silhouette exposing cutouts of a Japanese woman bound up in rope, plays out across the shoji screen. The paper becomes boundary and barrier between the voyeur on one side of the screen, and elusive subject behind.

This use of cultural imagery to draw in the viewer exploits Bruner's iconic knowledge. Well known cultural icons play on fascination with seemingly exotic cultures and entice the viewer to fantasize and project themselves to distant places. With imagery alone, the viewer remains observer, at best voyeur, and ultimately does not pierce the boundary, cultural or physical, put in place by the shoji screen. 
Through the use of sound, the work comes out beyond the plane of the image into the exhibition space. Quadraphonic sound panning is used to spatialize the sound emanating from different parts of the image, unwrapping the photographed body in space. The sonic properties described above physicalize not just the sound, but ultimately the image that is the source of the sound.

The process is a dual one, the sonification of visual imagery, and the resulting physicalization of the whole. The panes of the shoji screen serve to divide the image up into zones, each representing an independent point in both frequency and space. The section of the image behind each pane is sonified by a free running left-to-right scan. Each row of panes represents a range of frequencies, and the luminosity of image translates to the intensity of sound at a specific frequency in that band. These frequency ranges are distributed vertically in a series of non-overlapping bands that together cover the range of human hearing from $20-20,000 \mathrm{~Hz}$. The four rows of the shoji are in effect assigned, from bottom to top, to sub-low bass frequencies, middle-low frequencies, middle-high frequencies, and high treble frequencies. The Cartesian distribution of panes, vertically, and horizontally, situate the sound of each pane in quadraphonic space. A four channel sound system is arranged vertically in the plane of the image, creating spatial sound giving a sensation of height of the sound source. The high frequencies would thus emanate from the upper speakers while the lows from the speakers closer to the floor. The panes towards the left of the image are panned towards the left speakers, and the right panes correspondingly to the right speakers.

As the viewer moves in front of the image, a part of the image onscreen is cut out in the form of the moving silhouette, inverting the photo negative to the original positive. With movement, the viewer is able to uncover and expose parts of the provocative image with their own body. The dynamic visual form translates to an evolving soundscape that is projected into the exhibition space over the quadraphonic sound system. The exposed positive is brighter, and produces more sound than parts of the image left in negative. We can nearly trace the movement of the viewer by the movement of sound across the speaker space. The frequencies played are a direct function of the visual information in the photograph. The flowing curves of the subject's shoulders give melodic glissandi, while distinct entities like the eyes create gentle articulated envelopes of tone. Clearer vertical lines, such as the ropes binding the subject produce sharp percussive transients across a broad range of frequencies. The spiral pattern on ropes nuance this by smearing the percussive whip-like strike by "zipper" effects in time and frequency.

The congruence of what is seen and what is heard create a form of evoked synesthesia. The size of the projection creates a larger than life scale, while the undulating sound takes on physical properties, filling and pulsing in the volume of the gallery space. In a way very similar to the haptic feedback that physicalized sound gives the BioMuse performer, here the physicalization of sound goes beyond simple sonification of photographic image to create for the gallery visitor a tangible sensation of the process of uncovering done by their presence in front of Araki's photograph. Given that the perturbations in the image are the result of interaction between viewer presence and the original photograph, we can imagine a corporeal relationship between the voyeur's body as captured by infrared heat and the photographic subject in bondage that is rendered visceral and potentially carnal. With this, the work shifts modes from iconic knowledge to embodied, enacted experience.

\section{Coupling}

Dourish coins the notion of coupling to describe the transmission of embodied experience from one entity to another. Coupling for Doursh is a way in which the intentionality of one party is made 
meaningful to others. He cites Dennett's concept of intentional stance to describe coupled intentionality as a mutual achievement of observer and observed [Dennett 1987]. This is one way to view the transmission of intimate, visceral experience from performer to audience with the BioMuse, and from photographer to spectator via photographic subject in the case of Bondage. In these works, coupling takes place at two levels. First a classic process of physical media transduction physicalizes original gesture or image through the medium of sound into physical impulsions of air in space. This becomes the conduit by which expressive intentionality of the performer or photographer are transmitted to, coupled with, and felt by the spectator.

Through this process, the original intentionality of the artist is shared through a process of intimate, felt experience instead of rational analysis. It is here that artistic applications of concepts of embodied interaction and enactive knowledge can even invert relationships outlined by Dourish and Bruner.

Bruner presents enactive knowledge, in being action based, as the more primitive form of knowledge next to the imaged-based and language-based forms of iconic and symbolic knowledge. In a musical example such as the BioMuse, we follow this to affirm that a visceral, enactive knowledge of the EMG instrument and the sounds it is programmed to produce, must be established before semantic, structural musical understanding can be broached. With Bondage, we invert this, and take up Araki's play on the power of image in popular culture to use iconic knowledge as an entry point to entice the viewer to enter into a work in which enactive representation places them in intimate contact with an imaginary subject of desire. In both cases, the physical relationships into which the spectator's own body is immersed become the field of enactive sensation and understanding.

Dourish evokes Heidegger's concepts of zuhanden (ready-to-hand) and vorhanden (present-athand), ready-to-hand being the case of acting through something, and present-at-hand being the case where something becomes the object of attention [Heidegger 1927]. In interaction design, Dourish describes a tendency for a tool to go from ready-to-hand to present-at-hand. For the interaction designer, the interface is not seen only as a transparent extension of its user, but can draw the focus of attention of the user. In musical performance, it can be argued that the opposite is true. Musical instrument performance at first tends to draw focus and attention to the instrument and techniques for playing it. This is true of both performer and listener in early encounters with an instrument or with discovery of a new kind of music. With time (and some would argue virtuosity), the instrument increasingly becomes transparent, and is a natural extension of the performer's musical intentionality. In this case, the musical instrument has gone from present-at-hand to readyto-hand, the opposite trajectory to that of interactive interfaces. In media art, inasmuch as interactive technologies are used, interaction mechanisms can follow either trajectory. In the work presented here, they follow the musical instrument model - while the EMG sensing of the BioMuse, or the inverse FFT sonification of Bondage might first draw attention of the spectator trying to gain a base understanding of the mechanisms in play, the physicalization immerses them in sound and image where instrument and process can be forgotten, and hopefully become transparent. Through this, both BioMuse and Bondage invite the beholder to enter into an intimate space of sound, image, and felt experience. 


\section{References}

D. Bishop (2009) "Visualising and physicalising the intangible product: "What happened to that bloke who designed the marble answer machine?" Proceedings of the 3rd International Conference on Tangible and Embedded Interaction (TEI).

B. Bongers, B. (1998) “An interview with Sensorband”, Computer Music Journal 22, 13-24.

J. Bruner (1966). Toward a theory of instruction. Belknap Press of Harvard University Press (Cambridge MA).

C. De Boeck and Y. De Mey. Staalhemel. http://www.staalhemel.com Accessed 31 May 2011.

P. Carter (2004) Material Thinking: The Theory and Practice of Creative Research. Melbourne University Press (Melbourne).

D. Dennett (1987) The Intentional Stance. MIT Press (Cambridge MA).

E. A. Di Paolo, M. Rohde, H. De Jaegher (2010) Horizons for the Enactive Mind: Values, Social Interaction, and Play. In J. Stewart, O. Gapenne and E. A. Di Paolo (eds), Enaction: Towards a New Paradigm for Cognitive Science. Cambridge, MA: MIT Press, pp. 33 - 87.

P. Dourish (2004) Where the Action Is: The Foundations of Emborided Interaction. MIT Press (Cambridge MA).

Enactive Interfaces http://www.enactivenetwork.org Accessed 31 May 2011.

H. Fletcher and W. A. Munson (1933) "Loudness, its definition, measurement and calculation". Journal of the Acoustical Society of America, Vol 5, 82-108.

M. Heidegger (1927, transl. 1962) Being and Time. Harper \& Row (New York).

H. Helmholtz (1954/1877) On the Sensations of Tone as a Physiological Basis for the Theory of Music. Dover Publications (New York).

A. Kendon (2004) Gesture: Visible Action as Utterance. Cambridge University Press (Cambridge).

D. J. Levitin (2008) This is Your Brain on Music: Understanding a Human Obsession. Atlantic Books.

H. S. Lusted and R. B. Knapp (1996) "Controlling Computers with Neural Signals”, Scientific American, 275 (4) $82-87$.

A. Misra , G. Wang , P. R. Cook (2005) "SndTools: Realtime Audio DSP and 3D Visualization," Proceedings of the 2005 International Computer Music Conference (ICMC).

J. R. Pierce (1992) The Science of Musical Sound. Scientific American Library (New York).

V. Spolin (1999) Improvisation for the Theater: A Handbook of Teaching and Directing Techniques. Northwestern University Press (Chicago).

A. Tanaka (1993) "Musical technical issues in using interactive instrument technology with application to the BioMuse" Proc. Intl Computer Music Conference (ICMC).

A. Tanaka (2008) "Visceral Mobile Music Systems” In R. Adams, S. Gibson, S Müller Arisona, (eds.) Transdisciplinary Digital Art: Sound, Vision and the New Screen. (Springer).

A. Tanaka (In Press) "The Sound of Photographic Image" Sonicification, special issue of AI \& Society.

F. J. Varela, E. Thompson, E. Rosch (1999) The Embodied Mind: Cognitive Science and Human Experience. MIT Press (Cambridge MA). 